\title{
The Potential of LEDs in Plant-based Bio-pharmaceutical Production
}

\author{
Joey H. Norikane ${ }^{1}$ \\ Fraunhofer USA Center for Molecular Biotechnology, 9 Innovation Way, Suite 200, Newark, DE 19711
}

Additional index words. LED lighting, photo-recipes, plant-based biopharmaceuticals, transient protein expression

\begin{abstract}
Fraunhofer USA, Inc. Center for Molecular Biotechnology (FhCMB) has pioneered a transient plant-based biopharmaceutical platform to produce vaccines and therapeutics quickly and cost-effectively. Using this platform, plants are grown hydroponically in a controlled environment. The plants are vacuum infiltrated with a recombinant Agrobacteria carrying a plant viral-based hybrid vector and returned to a controlled environment for the target protein to be produced in infiltrated plant tissue. When target protein levels peak in leaf tissue, the plants are harvested and homogenized, extract is clarified and the target protein is purified. There are multiple opportunities in this system for light-emitting diodes (LEDs) to be applied to optimize biomass accumulation and the production of target proteins. There is potential to develop specific photo-recipes to optimize plant growth and target protein production.
\end{abstract}

Plant-based biopharmaceuticals are gaining acceptance in the pharmaceutical industry. Fraunhofer USA, Inc. Center for Molecular Biotechnology is a nonprofit company that has a pilot plant facility to produce vaccines and therapeutics for clinical trials using plant-based transient expression technology. In this field of biopharmaceuticals, plants are used to express foreign proteins for vaccines and therapeutics, as opposed to mining naturally occurring components of the plant itself.

The path for plant-based biopharmaceuticals has been long with its beginnings in the late 1980s and early 1990s (De Muynck and Boutry, 2010; Fischer et al., 2013; Rybicki, 2010; Yusibov et al., 2011). Much of the early work focused on developing stable lines of transgenic plants that were engineered to express the target protein. Issues arose from the use of edible plants for vaccines, since the plants could enter the commercial food chain. Development of these types of plant host has since slowed (Rybicki, 2010). During this time, plant viral vectors were also developed that could infect plant hosts and produce the protein of interest. The limitation of this approach is the instability of the vector during replication. This was later overcome with the development of hybrid transient expression systems using Agrobacterium tumefaciens (Musiychuk et al., 2007; Rybicki, 2010).

In the mid-1990s, engineered plant cell cultures were also developed. The benefit of these systems is the similarity of the technology to the methods currently used in

Received for publication 14 Nov. 2014. Accepted for publication 19 Dec. 2014.

This work was only possible with the support of Rebecca Snow, Dave Wienner, Ryan Dayton, and all of our colleagues at Fraunhofer USA Center for Molecular Biotechnology. Special thanks to Dr. Moneim Shamloul and his team for their assistance infiltrating the plants.

This paper was presented as part of a Colloquium entitled "The Importance of Light Quality for High Value Plant Products" during 28-31 July 2014 at ASHS Annual Conference in Orlando, FL.

${ }^{1}$ Corresponding author. E-mail: joey.norikane@ fhcmb.org. pharmaceutical manufacturing. The advantage is there are regulatory guidelines that are already in use making the path to a commercial product potentially more accessible (Rybicki, 2010). The engineering of plant cell cultures is hampered by long development lead times and the high cost of scaled manufacturing facilities.

There are a number of biopharmaceutical companies that use these technologies from pilot plant to commercial scale. A short list of these companies includes Icon Genetics (Germany), which uses transient expression and transgenic plants (http://www.icongenetics. $\mathrm{com} / \mathrm{html} / \mathrm{home} . \mathrm{htm})$, Kentucky BioProcessing (United States), also works with transient expression technology and transgenic plants (http://www.kbpllc.com/), and Protalix Biotherapeutics (Israel), which uses genetically engineered plant cell cultures (http://www. protalix.com/index.asp). Medicago USA uses transient expression technology and transgenic plants (http://www.medicago.com/). As an indication of the growing importance of plant-based biopharmaceutical business, Medicago was purchased by Mitsubishi Tanabe in July 2013 for \$357 million (Medicago, 2013). Caliber Biotherapeutics (United States) applies transient expression technology and operate a commercial scale facility in Texas (http://www.caliberbio.com/). FhCMB has pioneered transient expression technology and operates both an R\&D and pilot scale facility (www.fhcmb.org). This is a short list, but these companies and a growing number of others represent an industry that is maturing. The majority of the companies listed employ transient expression systems and a description of this technology follows.

\section{TRANSIENT EXPRESSION SYSTEMS}

There are multiple platforms for the production of biopharmaceuticals using plants. Transient expression systems have gained notoriety due to low cost, quick response time, and ability to produce a broad array of target proteins. The advantage of multiple target protein capability is a variety of products may be produced in a single facility. There are four common components of transient expression systems: 1) controlled environment biomass production, 2) recombinant Agrobacteria hybrid vectors, 3) vacuum infiltration for hybrid vector delivery, and 4) biomass harvest and protein purification. Each company has developed proprietary approaches for production. A general discussion of each component will give insight into the operation.

\section{Controlled environment biomass production}

One of the main mantras of pharmaceutical production is controlling all aspects of the production process recognizing that the final product will ultimately be injected into a person's arm. The processes, and requisite documentation, are developed and scaled to ensure the safety and efficacy of the final product. Process control begins with the plants, more specifically, plant seeds. There are a variety of plants both wild-type and genetically modified that are used in the production of plant-based biopharmaceuticals. In both cases, seed origin, genetic identification, genetic stability, among other characteristics of the seed line require extensive testing and documentation (Fischer et al., 2012). The biomass production process, in terms of nutrient solution (water source and fertilizer), light intensity, photoperiod, temperature, humidity, etc., should be well-defined. If LED lighting is applied, then additionally, the spectral quality of the light will need to be documented. These steps are taken to ensure the safety and efficacy of the final product. In the transient expression system, in addition to the plants, recombinant Agrobacteria hybrid vectors are needed to convert the plants into bioreactors producing the target protein.

\section{Recombinant agrobacteria hybrid vectors}

To initiate the conversion process, the plants' cell walls need to be breached and the appropriate gene expression machinery inserted into the cell. A hybrid vector is genetically engineered with Agrobacterium and plant viral vector components (Musiychuk et al., 2007). Agrobacterium establishes 
a pathway through the plants' cell walls and allows the viral vector to enter the cell. The viral vector typically contains replication and cell-to-cell movement machinery, in addition to the target protein. Once inside the cell, copies of the vector are replicated and begin to move systemically. Over a period of days, the target protein content in the plants' leaves peaks and the crop is ready for harvest. Hybrid vectors are an effective mean of producing protein in plant tissue, but to function, the vector has to be delivered in close proximity to the cell walls for the Agrobacterium to establish the entryway. This inoculation process is achieved through vacuum infiltration.

\section{Vacuum infiltration}

The method of applying a vacuum to leaf tissue in water has been used to measure the volume of the intercellular spaces in $\mathrm{C}_{3}$ and $\mathrm{C}_{4}$ plants (Byott, 1976). The vacuum infiltration approach has also been demonstrated as a means to introduce hybrid vectors into plant tissue for protein expression (Kapila et al., 1997). In this method, a vessel containing a diluted hybrid vector inoculum is placed in a vacuum chamber. The plants are inverted and submerged into the inoculum and a vacuum is drawn. As the pressure decreases, the air in the intercellular spaces in the leaves expands to the point where the air begins to exit the plant tissue. Continuing to decrease the pressure causes the air exiting the tissue to break from the exterior of the leaves and rise to the inoculum surface. When the vacuum is broken, the remaining intercellular air returns to a decreased volume at standard atmospheric pressure in the leaf tissue. As the remaining air in the leaf tissue returns to a decreased volume, the inoculum is drawn into the intercellular space. Vacuum infiltration is a scalable process that allows for the efficient and uniform inoculation of multiple plants with the hybrid vector. After inoculation, the additional weight of the inoculum causes the plants to bend and droop, but within several hours the plants recover and begin to transition into bioreactors for the target protein.

\section{Biomass harvest and protein purification}

After 4 to $8 \mathrm{~d}$, target protein production peaks in the leaf tissue. At this time, the aerial biomass is harvested from the trays, diced into smaller pieces, and mixed with extraction buffer. Once the entire crop is harvested and mixed, the remaining downstream processing steps are closed, i.e., the plant/buffer mixture is pumped through the remaining processes of homogenization, centrifugation, clarification, and filtration. The final steps are bulk chromatographic purification. The bulk drug product can be processed further, e.g., sterile bottling, etc., depending on the final purpose of the material.

\section{Transient expression with green fluorescent protein}

Figure 1 contains a time course of green fluorescent protein (GFP) accumulation in plants infiltrated with a hybrid vector with

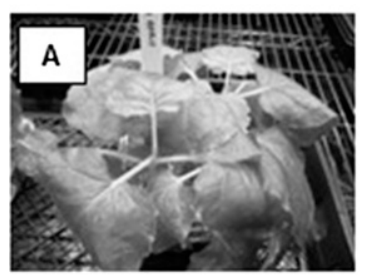

After Infiltration

$0 \mathrm{DPI}$
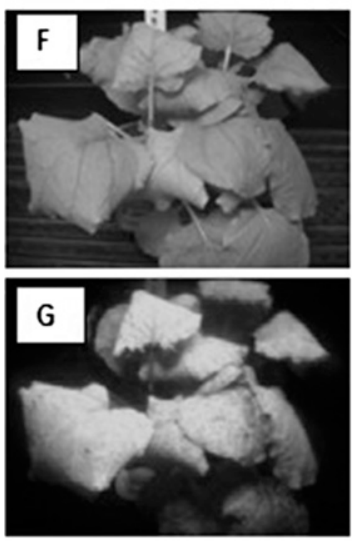

$5 \mathrm{DPI}$
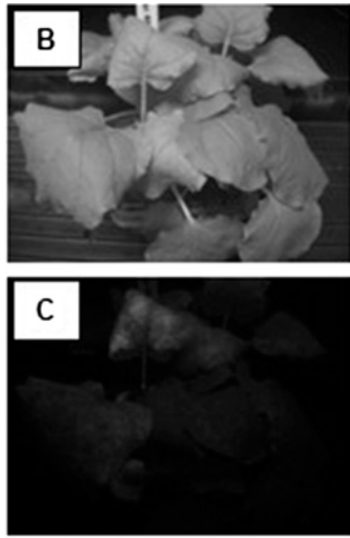

3 DPI
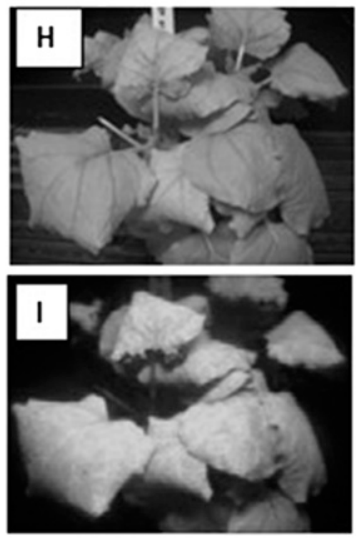

$6 \mathrm{DPI}$
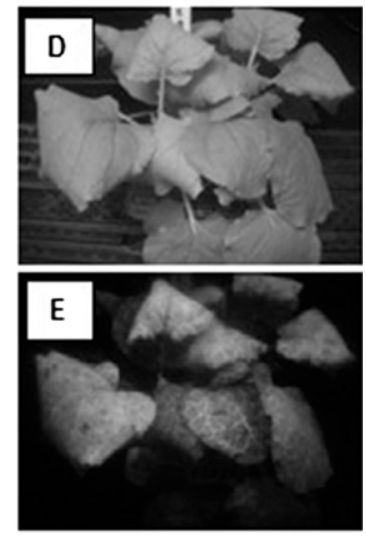

$4 \mathrm{DPI}$
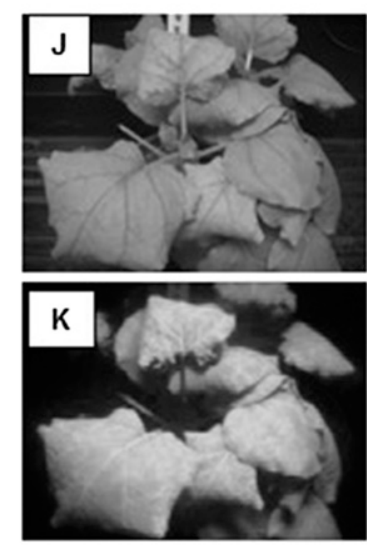

$7 \mathrm{DPI}$

Fig. 1. Time-course of green fluorescent protein (GFP) expression in Nicotiana benthamiana after vacuum infiltration. After vacuum infiltration (A) the plants appear wet and heavy. There is negligible fluorescence in the first few days post infiltration (DPI) and this time-course begins 3 DPI. The daily images are illuminated with fluorescent lights $(\mathbf{B}, \mathbf{D}, \mathbf{F}, \mathbf{H}, \mathbf{J})$, and with ultraviolet light $(\mathbf{C}, \mathbf{E}, \mathbf{G}, \mathbf{I}, \mathbf{K})$.

GFP as the target protein. Green fluorescent protein is derived from jellyfish and is widely used as a biomarker in plants (Taiz and Zeiger, 2006). Immediately after vacuum infiltration [0 days post infiltration (DPI)], the plant emerges wet and heavy, but recovers to a relatively normal appearance within several hours (Fig. 1A). Three days later (3 DPI), the plant looks normal with mild symptoms of leaf curl and slight yellowing (Fig. 1B). At this time, the accumulation of GFP in the leaf tissue begins to appear as spots and patches under ultraviolet light (Fig. 1C). During the prior days, the hybrid vector was working through the cell walls and engaging the plant's protein production system and the GFP needs to accumulate to a sufficient level to become visible. Figure 1D shows an increase in yellowing and the continued presence of leaf curl. More GFP has accumulated and the intensity of the fluorescence 4 DPI continues to increase (Fig. 1E). The symptoms become more pronounced over 5-7 DPI (Fig. 1F, H, and J). By 5 DPI (Fig. 1G), GFP has spread over much of the leaf tissue of the plant. On 6-7 DPI, the intensity of GFP signal appears to peak and the majority of the plant leaf tissue contains GFP (Fig. 1I and K). Over this time course, it is noted that the plant shows severe symptoms and produces a foreign protein, but the plant tissue has not become necrotic after 7 DPI.

\section{Adding value}

Green fluorescent protein can be transiently expressed in plants, but there is limited commercial value in producing GFP. The economics of biopharmaceuticals vary widely depending on the product. The value calculation has an array of factors that need to be considered. As a hypothetical exercise, a simplified scenario of adding value to a batch of plants for biopharmaceutical production follows. Suppose a client desired a vaccine to be produced at $\$ 1$ per dose. The protein of interest, i.e., the vaccine, is genetically engineered into an Agrobacterium-based hybrid vector. Several of the hybrid vector constructs are screened using a small number of plants for protein expression level and time to peak. At the end of screening, a construct is selected for further scale-up.

At the next level, biomass in the 1 to $5 \mathrm{~kg}$ range is used for process development. In this example, assume that the vaccine is expressed in plants at $200 \mathrm{mg}$ per $\mathrm{kg}$ of harvested biomass. This is the expression level as measured in the crude extract of plant biomass in a buffer. The purification of 


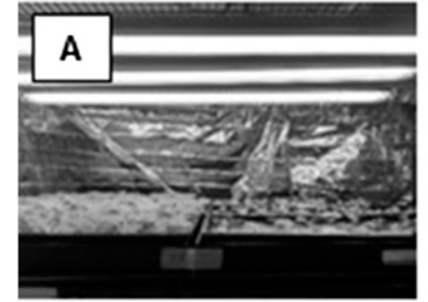

Fluorescent

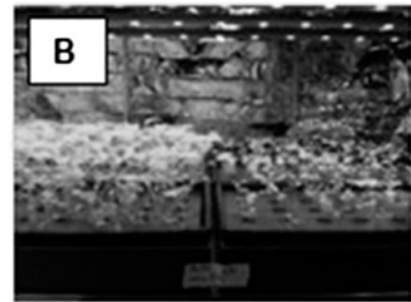

$\mathrm{R} / \mathrm{Fr}=1.2$

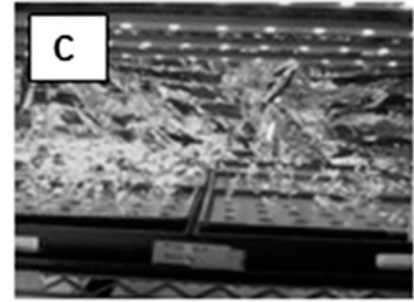

$\mathrm{R} / \mathrm{Fr}=0.7$

Fig. 2. Red $(660 \mathrm{~nm})$ and far red $(730 \mathrm{~nm})$ ratio treatments with fluorescent light (control). In each image, the Nicotiana benthamiana plants on the left are 4 weeks old and the plants on the right are 3 weeks old. The images are the control plants with fluorescent light (A), the red:far red (R:Fr) 1.2 ratio treatment (B), and the $\mathrm{R}: \mathrm{Fr}=0.7$ ratio treatment $(\mathbf{C})$. The stem elongation in both far red treatments was pronounced. There were germination issues for the $\mathrm{R}:$ Fr $=0.7$ treatment along with the elongation.

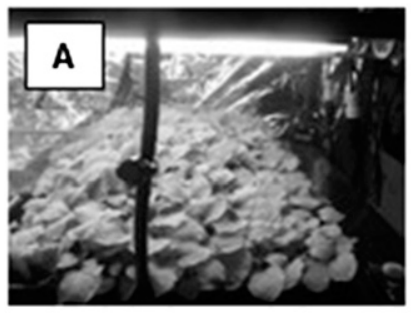

Fluorescent

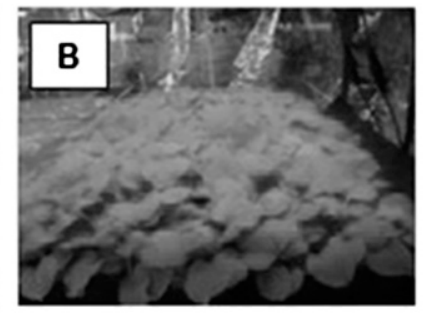

Red/Blue $=2.7$

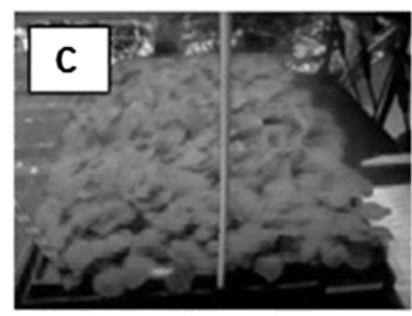

Red/Blue $=0.7$

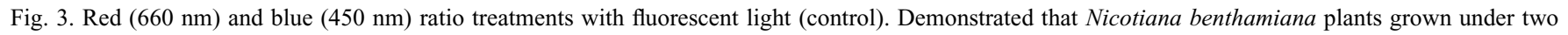
different LED treatments, red:blue $(\mathrm{R}: \mathrm{B})=2.7(\mathbf{B})$ and $0.7(\mathbf{C})$, were morphologically similar to the control plants under fluorescent lighting $(\mathbf{A})$.

the crude extract to isolate a human vaccine will incur a loss of protein. So, further assume that a purification process is developed that yields $100 \mathrm{mg}$ per $\mathrm{kg}$ harvested biomass. The purified protein at this point in the process can be used in a dose-ranging animal study to begin accumulating the necessary test results and documentation for an Investigational New Drug filing with the FDA for a Phase I human clinical trial. The results of the dose-ranging study would be used to help determine the effective dose for humans depending on the model of the animal study.

If the effective human dose was determined to be $10 \mu \mathrm{g}$ and the protein of interest is purified at $100 \mathrm{mg}$ per $\mathrm{kg}$ harvested biomass, then $100 \mathrm{~kg}$ of biomass will yield an estimated $10 \mathrm{~g}$ of purified product. The $10 \mathrm{~g}$ of product will then be divided into 1 million doses at $\$ 1$ per dose. So, $100 \mathrm{~kg}$ of biomass would have the value of $\$ 1$ million. But, there are significant embedded costs, in terms of time and money, for hybrid vector development and optimization, expression screening of multiple constructs, purification scale-up and process development, animal studies, and technology transfer to Good Manufacturing Practices pilot plant (Fischer et al., 2012).

\section{Potential of LED technology}

As plant-based biopharmaceutical production systems continue to evolve, there are opportunities for incorporating beneficial technology into the systems. The advantages of LED technology, in terms of energy efficiency, narrow-band photosynthetically active radiation $(P A R)$ output, extended operational life, etc., have a great deal of potential impact on the cost of production (Massa et al., 2008; Morrow, 2008). The production objective is simply to maximize target protein yields per kilogram of plant biomass. In the FhCMB case, using wild-type Nicotiana benthamiana the specific need is to maximize the leaf-to-stem ratio, since the target protein is expressed in leaves, as opposed to stems (Fig. 1K). Specific wavelengths of light can be used to alter a variety of plant morphological characteristics including leaf-to-stem ratio and flowering (Folta and Childers, 2008).

For example, if more target protein was produced from plants that were flowering before infiltration, then a preinfiltration process would be developed to achieve this condition. In a conventional greenhouse production setting with a day-neutral plant, i.e., a plant whose flowering is insensitive to daylength (Taiz and Zeiger, 2006), a growth regulator would be applied to the crop that would induce flowering. The entire crop would uniformly be flowering and ready for infiltration. But, this crop is raw material for a biopharmaceutical product for human application. The FDA will require the manufacturer to demonstrate that whatever is added to the process is removed from the final product. The safety of the growth regulator used in terms of the "allowable limit" that could be injected into a human being would have to be determined and clearance metrics developed that would satisfy regulatory concerns. Here the advantage of LED technology is a specific lighting regime could be applied that will effectively replace the growth regulator (Folta and Childers, 2008). Perhaps, the far red wavelength $(730 \mathrm{~nm})$ of light that is outside of the PAR 400-700 nm region could regulate photomorphogenic processes eliminating the need for traditional growth regulators and the associated regulatory issues.
A flexible LED lighting system could be used to develop optimized lighting strategies for $N$. benthamiana and other host plants. $N$. benthamiana is recognized as the model plant for transient protein expression (Goodin et al., 2008), but other plants have been/could be considered as well (Green et al., 2009). The previously stated goal is to maximize protein yields from a kilogram of biomass. Wild-type plants reduce the need for containment, but controlled environment agriculture systems can be designed and/or adapted for containment if genetically modified plants were used. If the use of a modified organism in a transient expression system was to yield significantly higher amounts of target protein, appropriate production systems would be developed and installed. Regardless of whether wild-type or genetically modified plants are used, the ability to optimize light quality and develop photo-recipes for the organisms at the various developmental stages with LED technology is beneficial.

\section{LED applications}

In the FhCMB application, plants are grown as a raw material for biopharmaceutical production. This application is different from other horticultural enterprises where flowering, canopy size, and nutritional content, among others, are the primary production driving characteristics. For plant-based biopharmaceuticals, understanding that the target proteins are produced in the leaf tissue, lead to the exploration of maximizing the leaf-to-stem ratio with a dimmable, four-channel [blue $(450 \mathrm{~nm})$, green $(525 \mathrm{~nm})$, red $(660 \mathrm{~nm})$, and far red $(730 \mathrm{~nm})]$ LED lighting system.

Ratio of red and far red light. Far red light in the range of $730 \mathrm{~nm}$ is well-known to have an impact on plant growth and development. 
Particularly, the red (660 nm):far red (R:Fr) ratio can induce a shade plant response leading to leaf expansion and stem elongation among other changes. The potential of using light to increase the leaf-to-stem ratio, which would then enhance target protein yields per kilogram of biomass, requires further investigation.

The overall light levels were equivalent among the fluorescent control, $\mathrm{R}: \mathrm{Fr}=1.2$, and $\mathrm{R}: \mathrm{Fr}=0.7$ treatments. Figure 2A shows the fluorescent control $N$. benthamiana plants 4 weeks old on the left and 3 weeks old on the right. Figure 2B shows the $\mathrm{R}: \mathrm{Fr}=1.2$ plants with elongated stems for both the 3 weeks (on right) and 4 weeks (on left) sets of plants. Figure $2 \mathrm{C}$ is of the $\mathrm{R}: \mathrm{Fr}=0.7$ plants where similar stem elongation is apparent, but also there was low germination in this light treatment. The results were that the addition of far red light induces stem elongation in both the R:Fr treatments. At R:Fr $=0.7$, the higher amount of far red light inhibited seed germination. The conclusion was with these $\mathrm{R}:$ Fr treatments, there was a decrease in the leaf-to-stem ratio.

Red and blue light. The application of far red light resulted in the reduction of the leafto-stem ratio, so LED evaluation efforts were directed to a more basic question. The next research objective was to evaluate the biomass produced using commercially available LED red $(660 \mathrm{~nm})$ to blue $(450 \mathrm{~nm})$ ratios and compare the results to plants grown under fluorescent lighting. The two red:blue (R:B) ratios chosen were 2.7 and 0.7 , respectively. Figure 3 shows slight differences in canopy heights among the fluorescent control and the two R:B treatments with the LED plants being shorter, but there were no significant differences (data not shown). There is a broad array of LED lighting systems for horticultural applications making further evaluations prudent.

Potential of LED lighting systems in production. LED lighting systems offer an opportunity to customize the light environment for each species to maximize a broad array of plant characteristics. With a dimmable, multiple channel, e.g., red, blue, green, far red, and others, programmable system there is potential to optimize the environment for germination and survival. Once the seedlings are established, there is a programmed transition of the lights for maximum vegetative growth by modifying the light spectra, light intensity, and photoperiod. If flowering is desired, when appropriate, the lighting system could then be programmed to transition to a flowering configuration. Specific LED photo-recipes for individual crops could be developed that would optimally address each stage of plant growth to maximize production and value-added characteristics.

\section{SUMMARY}

Plant-based biopharmaceuticals are gaining acceptance as an effective and viable commercial technology. Food and Drug Administration-approved Phase I and II human clinical trials of plant-based biopharmaceutical products have been completed demonstrating safety and efficacy. Light is a critical component for the production of these vaccines and therapeutics. With LED lighting there is potential to combine energy efficiency and spectral specificity to optimize the plants' light environment at each stage of growth and development. The driving metric for plants used in transient expression systems is protein production per kilogram of plant biomass. Further research is needed, but there is potential for LED lighting systems to deliver optimized photo-recipes to increase production and decrease operational costs.

\section{Literature Cited}

Byott, G.S. 1976. Leaf air space in $C_{3}$ and $C_{4}$ species. New Phytol. 76(2):295-299.

De Muynck, C.N. and M. Boutry. 2010. Production of antibodies in plants: Status after twenty years. Plant Biotechnol. J. 8:529-563.

Fischer, R., S. Schillberg, J.F. Buyel, and R.M. Twyman. 2013. Commercial aspects of pharmaceutical protein production in plants. Curr. Pharm. Des. 19:5471-5477.

Fischer, R., S. Schillberg, S. Hellwig, R.M. Twyman, and J. Drossard. 2012. GMP issues for recombinant plant-derived pharmaceutical proteins. Biotechnol. Adv. 30:434-439.

Folta, K.M. and K.S. Childers. 2008. Light as a growth regulator: Controlling plant biology with narrow-bandwidth solid-state lighting systems. HortScience 43:1957-1964.

Goodin, M.M., D. Zaitlin, R.A. Naidu, and S.A. Lommel. 2008. Nicotiana benthamiana: Its history and future as a model for plant-pathogen interactions. Mol. Plant Microbe Interact. 21(8):1015-1026

Green, B.J., M. Fujiki, V. Mett, J. Kaczmarczyk, M. Shamloul, K. Musiychuk, S. Underkoffler, V. Yusibov, and V. Mett. 2009. Transient protein expression in three Pisum sativum (green pea) varieties. Biotechnol. J. 4:1-8.

Kapila, J., R. De Rycke, M. van Montagu, and G. Angenon. 1997. An Agrobacterium-mediated transient gene expression system for intact leaves. Plant Sci. 122:101-108.

Massa, G.D., H-H. Kim, R.M. Wheeler, and C.A. Mitchell. 2008. Plant productivity in response to LED lighting. HortScience 43:1951-1956.

Medicago. 2013. Medicago announces agreement to be acquired by Mitsubishi Tanabe Pharma in a transaction values at $\$ 357 \mathrm{M}$. $<\mathrm{http}: / / \mathrm{www}$. medicago.com/files/documents_news/Press $\%$ 20Release \%20Medicago\%20FINAL\%20EN. $\mathrm{pdf}>$.

Morrow, R.C. 2008. LED lighting in Horticulture. HortScience 43:1947-1950.

Musiychuk, K., N. Stephenson, H. Bi, C.E. Farrance, G. Orozovic, M. Brodelius, P. Brodelius, A. Horsey, N. Ugulava, A-M. Shamloul, V. Mett, S. Rabindran, S.J. Streatfield, and V. Yusibov. 2007. A launch vector for the production of vaccine antigens in plants. Influenza Other Respi. Viruses 1:19-25.

Rybicki, E.P. 2010. Plant-made vaccines for humans and animals. Plant Biotechnol. J. 8:620-637.

Taiz, L. and E. Zeiger. 2006. Plant physiology. 4th ed. Sinauer Associates, Inc., Sunderland, MA.

Yusibov, V., S.J. Streatfield, and N. Kushnir. 2011. Clinical development of plant-produced recombinant pharmaceuticals: Vaccines, antibodies and beyond. Hum. Vaccin. 7(3):313321. 